\title{
La importancia de comenzar a investigar el rol del establecimiento de conexiones entre enunciados en la comprensión del discurso oral expositivo
}

\section{The importance of studying the role of establishing discourse connections in the comprehension of expository oral discourse}

\author{
Jazmín Cevasco \\ Consejo Nacional de Investigaciones Científicas y Técnicas - Universidad de Buenos Aires, Argentina
}

Disponible online 30 de abril de 2014

\begin{abstract}
El objetivo de este trabajo es señalar las aportaciones que los estudios acerca de la comprensión del discurso escrito expositivo, el discurso oral no expositivo y el discurso oral expositivo pueden hacer a la promoción del aprendizaje en el ámbito educativo. A su vez, destacar la importancia de realizar nuevos estudios que consideren las variables que facilitan la comprensión del discurso escrito expositivo, y no se han investigado en relación con el discurso oral expositivo. Con este fin, se presentan estudios acerca del diseño de textos de refutación y procedimientos de revisión de textos en la comprensión del discurso escrito expositivo. A su vez, se presentan estudios acerca del procesamiento de errores del habla, claves prosódicas, conexiones causales, y marcadores del discurso en la comprensión del discurso oral no expositivo. Finalmente, se presentan estudios acerca del rol de los marcadores del discurso, velocidad de dicción y entrenamiento en habilidades cognitivas en la comprensión del discurso oral expositivo. Se señala que la consideración de toda esta serie de estudios puede contribuir a decidir qué cambios introducir en los materiales didácticos escritos para facilitar la comprensión lectora, y qué variables acerca de la comprensión del discurso oral expositivo y no expositivo se puede tener en cuenta a la hora de exponer una clase.
\end{abstract}

Palabras clave: Discurso Expositivo; Comprensión; Discurso Oral.

The aim of this paper was to highlight the contribution of studies on the comprehension of expository written discourse, and expository and non-expository oral discourse to the facilitation of student learning. It also draws attention to the importance of conducting new studies to explore the role of the variables that facilitate expository written discourse and which have not been investigated in relation to expository oral discourse. Studies that have focused on the design of refutational texts and text revision procedures in the comprehension of expository written discourse are presented, as well as studies on the processing of disfluencies, prosodic cues, causal connections and discourse markers in the comprehension of non-expository oral discourse. Finally, studies on the role of discourse markers, speech rate and metacognitive strategy instruction are presented. It is suggested that these studies can contribute to deciding what revisions to make to written materials in order to improve reading comprehension, and what variables that facilitate the comprehension of expository and non-expository oral discourse to take into account when presenting a lecture.

Key words: Expository Discourse; Comprehension; Oral Discourse.

Correspondencia: Jazmín Cevasco. Consejo Nacional de Investigaciones Científicas y Técnicas, Instituto de Investigaciones, Facultad de Psicología, UNIVERSIDAD DE BUENOS AIRES. Av. Independencia 3065, 3º piso, of. 8. Buenos Aires (C1225AAM), Argentina. E-mail: jazmincevasco@ psi.uba.ar.

Se agradece a los revisores anónimos por sus comentarios y sugerencias, los cuales contribuyeron a mejorar este trabajo. 
La comprensión del discurso es una de las más complejas actividades cognitivas humanas, e implica el establecimiento de conexiones entre enunciados (Mulder y Sanders, 2012; Zwaan y Rapp, 2006). En particular, el establecimiento de conexiones causales permite generar inferencias, las cuales pueden definirse como la activación de información no mencionada explícitamente en el texto (Molinari Marotto, Barreyro, Cevasco y van den Broek, 2011; Trabasso y Sperry, 1985; van den Broek, 2010).

Uno de los objetivos de la investigación en psicolingüística es establecer cómo puede facilitarse la comprensión del discurso expositivo, dado que representa una herramienta fundamental para el aprendizaje, y que su procesamiento supone un desafío mayor que el de otros tipos de discurso (Cromley, Snyder-Hogan, y Luciw-Dubas, 2010; Kendeou y van den Broek, 2005, 2007). En consecuencia, es importante explorar qué cambios puede introducirse tanto en los materiales didácticos de discurso escrito expositivo, así como en el modo en el que los profesores exponen discurso oral expositivo durante sus clases, a fin de facilitar la comprensión del estudiante.

Con el fin de explorar qué aportes pueden hacer los estudios realizados a este objetivo, se presentarán tanto investigaciones acerca de la comprensión del discurso escrito expositivo, así como acerca del discurso oral no expositivo y expositivo, y se destacará la necesidad de realizar nuevos estudios que investiguen el rol de las variables que se ha encontrado que facilitan la comprensión del discurso expositivo escrito, y que no se han explorado en relación con el discurso oral expositivo.

Cabe resaltar que el establecimiento de conexiones entre enunciados en la comprensión del discurso oral no ha recibido la misma atención que en la comprensión del discurso escrito (Zwaan y Rapp, 2006). Esta brecha es importante, ya que ambos son necesarios para el desempeño en el ámbito educativo (Ratebi y Amirian, 2013; Rasouli, Mollakhan y Karbalaei, 2013), y que presentan características distintivas que podrían conducir a diferencias en su procesamiento (Fraundorf y Watson, 2011). Entre ellas, el discurso oral requiere ser procesado mientras es producido, y suele forzar a los hablantes a hablar aún cuando no están seguros de lo que quieren decir. En consecuencia, es común que corrijan o reformulen enunciados, produzcan pausas marcadas por "eh" o "este", o repitan palabras (Barr y Seyfeddinipur, 2010; Fox Tree y Schrock, 1999). Por otro lado, su comprensión se ve facilitada por la transmisión de información no verbal, tal como la entonación y velocidad de dicción con la que es producido un enunciado (Gunraj y Klin, 2012; Wagner y Watson, 2010). El discurso escrito, por su parte, permite al lector procesar el discurso al ritmo que elija, y re-leer u omitir secciones (Ferreira y Anes, 1994), pero no permite la transmisión de información no verbal (Chafe, 1994).

Con el fin de señalar qué herramientas pueden aportar las investigaciones acerca de la comprensión del discurso expositivo escrito a la facilitación de la comprensión en el ámbito educativo, se presentarán estudios acerca del diseño de textos de refutación y procedimientos de revisión de textos. A su vez, se describirán estudios acerca del discurso oral no expositivo, a fin de presentar lo que sabemos acerca de los procesos que están en juego cuando un estudiante escucha discurso oral, sea éste expositivo o no, tales como la necesidad de reparar errores del habla (correcciones, repeticiones y pausas marcadas) e interpretar claves prosódicas (límites prosódicos y acentos), así como de establecer conexiones causales entre enunciados, e integrarlos a partir de la presencia de marcadores del discurso. A pesar de que el tipo de material utilizado (instrucciones y conversaciones) presenta características que lo diferencian del discurso oral expositivo, se propone que el conocimiento acerca de estos procesos necesarios para comprender discurso oral en general, puede ser tenido en cuenta tanto por el docente que intenta facilitar la comprensión de sus estudiantes, como por el investigador que intenta estudiarla. Es decir, el discurso oral, expositivo o no, incluye la producción de errores del habla (Fox Tree, 1995) y la provisión de claves prosódicas (Ferreira y Anes, 1994), así como la necesidad de establecer conexiones de causa-efecto, e integrar enunciados adyacentes a partir de marcadores del discurso. Por su parte, se mencionan estudios acerca de la comprensión del discurso oral expositivo, los cuales han tendido a enfocarse en la velocidad de dicción del profesor, el rol de los marcadores del discurso, y el entrenamiento en habilidades metacognitivas en la comprensión de estudiantes universitarios no nativos del idioma de la clase. Aunque estos estudios no se han enfocado en la comprensión de estudiantes nativos, las variables que consideran forman también parte de los procesos que deben llevar a cabo estos estudiantes durante el dictado de clases, y pueden ser consideradas, en especial, en relación con los pre-universitarios, quienes se encuentran aún desarrollando su capacidad de comprensión.

\section{Comprensión del discurso expositivo escrito}

A continuación, se presentarán estudios acerca del efecto de introducir cambios en la estructura de los textos expositivos para facilitar su comprensión.

\section{Textos de refutación}

Los autores de textos expositivos suelen presuponer que el lector cuenta con conocimiento previo adecuado para establecer las conexiones necesarias para comprenderlos (Beck, McKeown, Sinatra, y Loxterman, 1991). Sin embargo, los lectores que tienen conocimiento previo incorrecto acerca del tema de un texto recuerdan menos información que los que poseen conocimiento correcto (Kendeou y van den Broek, 2005). Con el fin de superar estas limitaciones, se han diseñado los "textos de refutación". Éstos buscan enumerar las ideas previas incorrectas que suelen tener los lectores, refutarlas y presentar el conocimiento correcto. La activación conjunta de la idea errónea y la correcta promovería su integración, y daría lugar a una comprensión más profunda (Kendeou y van den Broek, 2007). El siguiente es un ejemplo de uno de estos textos (van 
den Broek \& Kendeou, 2008, adaptado de McCloskey, 1982): "La Mecánica Newtoniana puede ayudarnos a explicar eventos de nuestra vida cotidiana. Imagina esta situación: Si una persona sostiene una piedra a la altura de su hombro mientras camina, ¿qué pasará cuando la suelte? Mucha gente responde que caerá en el punto en el que fue soltada. Otra gente piensa que caerá detrás del punto en que sea soltada. En realidad, la piedra se moverá hacia adelante mientras cae, aterrizando delante del punto en el que fue soltada. La Mecánica Newtoniana explica esto del siguiente modo: cuando una piedra cae, continúa moviéndose hacia adelante a la misma velocidad a la que venía caminando la persona, porque ninguna fuerza actúa para cambiar la velocidad horizontal." (p. 340$)$

Diakidoy, Kendeou y Ioannides (2003) encontraron que estudiantes de escuela primaria respondían mejor a preguntas tanto inmediatas como demoradas, a partir de la lectura de un texto de refutación que uno expositivo clásico del tema "Energía". Mason, Gava y Boldrin (2008) encontraron que la respuesta a preguntas por parte de estudiantes de primaria se facilitaba cuando leían un texto de refutación del tema "Electricidad" que uno expositivo clásico, especialmente cuando encontraban interesante este tema. McCrudden (2012) encontró que estudiantes de escuela primaria que lograban modificar sus ideas incorrectas iniciales por el conocimiento correcto que introducía un texto de refutación sobre Ciencias Naturales, tendían a hacer más comentarios que indicaban que estaban evaluando su propia comprensión, y a generar más inferencias que conectaban el texto con su conocimiento previo, que los estudiantes que no lograban hacer estos cambios conceptuales.

Kendeou y van den Broek (2007) encontraron que la lectura de textos de refutación facilitaba la comprensión de estudiantes universitarios, ya que aquellos que tenían conocimiento previo incorrecto realizaban más comentarios, mientras leían, que indicaban que habían modificado su conocimiento incorrecto por el correcto, y leían las_oraciones que lo refutaban más lentamente a partir de este tipo de texto que del expositivo clásico. Otros estudios encontraron resultados similares con estudiantes universitarios (Ariasi y Mason, 2011; Broughton, Sinatra y Reynolds, 2010), en especial cuando reportaban concebir la construcción de conocimiento como un proceso dinámico que involucra cambios de punto de vista (Kendeou, Muis y Fulton, 2011).

\section{Procedimientos de revisión de textos}

Los procedimientos de revisión de textos involucran la realización de revisiones o cambios sistemáticos en el formato de los textos expositivos. Entre ellos, el procedimiento de repetición de términos se basa en el modelo de Kinstch y Van Dijk (1978). Propone reparar los cortes de coherencia (los cuales ocurren cuando un par de oraciones adyacentes no comparten términos), reescribiendo la oración siguiente al corte de forma que se repita una palabra de la anterior, reordenando las pala- bras de la segunda oración para que el lector encuentre primero los términos que ya ha leído y a continuación los nuevos, y haciendo explícita cualquier referencia implícita. Britton y Gulgoz (1991) encontraron un mejor recuerdo tanto inmediato como demorado de un texto histórico revisado en comparación con el texto original por parte de estudiantes universitarios. Vidal-Abarca, Martínez y Gilabert (2000) pidieron a un grupo de estudiantes de enseñanza secundaria que leyeran la versión original o la versión revisada de un texto de Historia, las recordaran por escrito (inmediatamente y una semana después), y respondieran a una serie de preguntas de inferencia. Estas preguntas requieren que el lector establezca conexiones entre oraciones contiguas y distantes. Los resultados indicaron un mejor recuerdo inmediato y demorado del texto revisado que el original, pero no mejores puntajes de respuesta a preguntas de inferencia. Resultados similares en cuanto a la facilitación del recuerdo del texto revisado fueron obtenidos con estudiantes de enseñanza secundaria por Gilabert, Martínez y Vidal-Abarca (2005).

Un segundo procedimiento de revisión es el de incremento de la densidad de relaciones. Éste se basa en un sistema computacional llamado Expository Text Analysis Tool (Vidal-Abarca et al., 2002), el cual propone clasificar las relaciones entre las ideas de cualquier texto expositivo en: inicio, razón, resultado, causa, ejemplo y descripción. Una vez que se ha establecido tanto el tipo como la cantidad de relaciones existentes entre las oraciones de un texto, la revisión consiste en añadir oraciones con las que las originales puedan establecer nuevas conexiones. Esto aumenta el promedio de relaciones por oración, y facilita la integración de oraciones distantes. Vidal-Abarca et al. (2002) encontraron que esta revisión facilitaba la respuesta de estudiantes de enseñanza secundaria a preguntas de inferencia, a partir de un texto de Biología. Barreyro, Molinari Marotto, Bechis y Cevasco (2012) encontraron a su vez una facilitación de la respuesta a preguntas de inferencia, esta vez por parte de estudiantes universitarios, que habían un texto de Biología revisado a través de este método, en comparación con un grupo que había leído la versión original.

En resumen, las investigaciones acerca del discurso escrito expositivo sugieren que la co-activación de oraciones que describen conocimiento incorrecto y las que introducen conocimiento correcto, la reparación de cortes de coherencia, y el agregado de oraciones con las que las originales puedan establecer nuevos vínculos facilitan la comprensión lectora. Sin embargo, no nos permiten establecer si se observaría el mismo efecto con respecto al discurso oral expositivo, ya que éste tiene características que lo distinguen del discurso escrito.

\section{Comprensión del discurso oral no expositivo}

En esta sección, se presentarán investigaciones acerca del procesamiento de errores del habla y claves prosódicas en el seguimiento de instrucciones, y estudios acerca del rol de las conexiones causales, marcadores del discurso y cambios de 
tema en la comprensión de conversaciones. Estos estudios se enfocan en los procesos que están en juego durante la comprensión del discurso oral, sea éste expositivo o no, y que forman parte de los procesos que un estudiante debe llevar a cabo para comprender una clase, por lo que pueden ser tenidos en cuenta por el profesor, a fin de facilitarlos.

\section{Procesamiento de errores del habla y claves prosódicas}

Estos estudios se han enfocado mayormente en la comprensión a nivel oración, y en el seguimiento de referencias. Es decir, en el efecto de escuchar errores del habla y claves prosódicas en la capacidad de los oyentes de reparar estos errores y segmentar los enunciados, pero no en el establecimiento de conexiones, tales como las causales, entre ellos. Sin embargo, sus resultados pueden proveer información importante para conocer los procesos que lleva a cabo un oyente cuando establece tales conexiones discursivas, ya que la reparación de los errores del habla, la segmentación de la señal de habla, y la identificación de las palabras más importantes a partir de claves prosódicas, son necesarias para comprender cada enunciado, y poder luego conectarlo con otros.

En cuanto a procesamiento de errores del habla, Fox Tree (1995) investigó el efecto de las correcciones y las repeticiones en la comprensión de instrucciones. Las correcciones ocurren cuando los hablantes abandonan el enunciado que han comenzado, para reformularlo. Por ejemplo, en "Y la próxima figura, tiene-se ve como un martillo". Las repeticiones ocurren cuando los hablantes repiten palabras o grupos de palabras "Y la próxima figura-figura se ve como un martillo". Utilizó una tarea de monitoreo de palabras. Para realizar esta tarea, se comunica a los participantes una determinada palabra que deben tener en mente, y se les presentan una serie de enunciados. Los oyentes deben presionar un botón cuando la escuchan. Se espera que los tiempos de reacción sean menores cuanto más integrado esté el discurso escuchado hasta que ocurre la palabra. En este estudio, se presentaron versiones originales o editadas del material. En las originales, los enunciados incluían la corrección o repetición. En las versiones editadas, los errores del habla habían sido recortados a través de un programa de edición. Los tiempos de reacción para identificar las palabras que ocurrían a continuación de una corrección fueron mayores, que para las mismas palabras presentadas sin la corrección. Los tiempos de reacción para las palabras que ocurrían a continuación de una repetición no mostraron diferencias entre versiones originales y editadas. Es decir, mientras las correcciones causaron algunas dificultades de procesamiento, las repeticiones no lo hicieron. Por otra parte, las repeticiones tampoco facilitan el recuerdo de palabras escuchadas después de ellas (MacGregor, Corley,y Donaldson, 2010).

Brennan y Schober (2001) observaron que los oyentes se recuperan más fácilmente de errores del habla que ocurren a media palabra y que contienen pausas marcadas por "eh" o "este" (tal como: "Mueve el cursor hacia el cuadrado amar-eh- violeta"), que de errores del habla que ocurren entre palabras ("Mueve el cursor hacia el cuadrado amarillo-violeta"), ya que son más capaces de identificar palabras de prueba que ocurren a continuación de los primeros. Otros estudios han encontrado que las pausas marcadas promueven que el oyente estime que el hablante tiene problemas de articulación, o va a referirse a información nueva, o difícil de describir (Barr, y Seyfeddinipurr, 2010; Corley y Hartsuiker, 2011).

Otra serie de estudios han investigado cuál es el rol de las claves prosódicas, tales como los límites prosódicos y acentos, en la capacidad de los oyentes de segmentar los enunciados que escuchan, y detectar qué palabras brindan información nueva. Los límites o quiebres prosódicos ocurren cuando los hablantes prolongan la duración de una palabra, y hacen luego una pausa (Kjelgaard y Speer, 1999). Se espera que esta pausa o quiebre facilite la segmentación del enunciado. Los acentos involucran incrementos en la duración e intensidad con que los hablantes producen segmentos del enunciado (Ladd, 1996), por lo que se espera que aumenten la atención del oyente hacia las palabras que introducen información nueva.

Schafer, Speer, Warren y White (2000) investigaron el rol de los límites prosódicos en la capacidad de los oyentes de segmentar enunciados escuchados durante una tarea de juego cooperativo, en la que los hablantes daban instrucciones que podían interpretarse de distintos modos, según cómo se las segmentara. Por ejemplo: "Cuando el triángulo gire (límite o quiebre prosódico) el cuadrado encontrará otra ficha" o "Cuando el triángulo gire el cuadrado (límite o quiebre prosódico) éste quedará en mejor posición”. Observaron que cuando el segmento ambiguo ("Cuando el triángulo gire") era presentado a un grupo de oyentes, éstos eran capaces de identificar el enunciado del que provenía, sugiriendo que habían podido utilizar los límites prosódicos.

Snedeker y Trueswell (2003) encontraron que cuando un grupo de hablantes daba instrucciones acerca de objetos en un tablero que podían interpretarse de distintos modos ("Toca la rana con la flor"), tendían a marcar límites prosódicos, y los oyentes podían seguir las instrucciones correctamente. Cuando los objetos dispuestos solo permitían una interpretación, el uso de claves prosódicas se veía reducido, y los oyentes no podían seguir las instrucciones correctamente.

Kraljic y Brennan (2005) pidieron a un grupo de hablantes que proveyeran instrucciones que podían ser ambiguas ("Ubica al perrito en la canasta sobre la estrella"), y les permitieron interactuar libremente con los oyentes. En este caso, tanto cuando los objetos dispuestos daban lugar a dos interpretaciones, como cuando daban lugar solo a una, los hablantes producían límites prosódicos, y los oyentes eran capaces de utilizarlos. Concluyeron que, al utilizar una tarea más interactiva, sus resultados se encuentran más cercanos a la comunicación cotidiana. Otros estudios han encontrado a su vez un rol facilitador de la segmentación de enunciados para los límites prosódicos (Bögels, Schriefers, Vonk, Chwilla, y Kerkhofs, 2010; Fraundorf, 
Watson y Benjamin, 2012).

En cuanto al efecto de acentuar palabras dentro de un enunciado, se han realizado estudios para examinar si los oyentes pueden identificar que se está introduciendo información nueva. Weber, Braun y Crocker (2006) pidieron a oyentes que siguieran pares de instrucciones. Cando la primera introducía un objeto a seleccionar en la pantalla de una computadora (tijeras violetas), y la segunda acentuaba información nueva de color (tijeras ROJAS), los oyentes miraban más rápidamente al objeto correcto que cuando acentuaban información ya mencionada (TIJERAS rojas), sugiriendo que podían utilizar el acento como clave de que se estaba señalando información nueva. Sedivy, Tanenhaus, Chambers y Carlson (1995) presentaron a oyentes dos objetos en un tablero que diferían en color (una taza verde y una taza azul), y pidieron a un grupo de hablantes que proveyeran instrucciones para seleccionarlos. Encontraron que cuando las instrucciones acentuaban la información nueva ("Toma la taza verde. Ahora toma la taza AZUL"), aumentaban las miradas de los oyentes hacia el objeto correcto (taza azul) en comparación con cuando no lo hacían.

Ito y Speer (2008) encontraron que oyentes que seguían instrucciones para decorar un árbol de Navidad, miraban más rápidamente al objeto correcto cuando los hablantes acentuaban la nueva información (como en "Primero, cuelga la estrella azul. Ahora, cuelga la estrella VERDE”) que cuando acentuaban información ya mencionada ("Ahora, cuelga la ESTRELLA verde"). Otras investigaciones han encontrado, a su vez, que la acentuación de palabras que introducen nueva información facilita el procesamiento de enunciados, a través de la medición de potenciales evocados (Toepel, Pannekamp, y Alter, 2007).

En resumen, estos estudios nos brindan información acerca del efecto de las correcciones, pausas marcadas, límites prosódicos y acentos en la capacidad de los oyentes de interpretar enunciados. Es decir, los hablantes (sean docentes o no), tienden a producir errores del habla, y claves prosódicas, los cuales necesitan ser reparados e interpretados por el oyente (estudiante o no). Considerar estos procesos puede ser útil para un profesor, a fin de tener en cuenta, por ejemplo, que si produce enunciados que pueden ser ambiguos será importante que realice pausas que indiquen cómo segmentarlos, y cuando introduce información nueva, que la acentúe para atraer la atención de los estudiantes hacia ellas. De no hacerlo, los estudiantes podrían no ser capaces de interpretar los enunciados correcta y rápidamente, y dificultarse el establecimiento de conexiones entre ellos, perjudicando la comprensión de la clase.

\section{Procesamiento de conexiones causales, cambios de tema y marcadores del discurso}

Algunos estudios preliminares se han enfocado en cómo se establecen conexiones entre enunciados en la comprensión de conversaciones, por lo que representan un primer paso para investigar si las variables que juegan un rol facilitador en la comprensión del discurso expositivo escrito pueden jugarlo también en la comprensión del discurso oral no expositivo. $\mathrm{Si}$ bien no utilizan materiales de discurso expositivo, exploran el rol de variables que también forman parte de la comprensión de este tipo de discurso, tales como el establecimiento de conexiones causales entre enunciados, el procesamiento de cambios de tema y la integración de enunciados adyacentes a partir de la presencia de marcadores del discurso.

Cevasco y van den Broek (2008) presentaron una sección de una entrevista realizada en programa de radio a dos grupos de estudiantes universitarios. A uno de ellos, se le pidió que la escuchara, escribiera todo lo que recordaba, y respondiera a una serie de preguntas. A un segundo grupo, se le pidió que leyera la transcripción, y realizara las mismas tareas. Utilizando los procedimientos propuestos por la Teoría de Red Causal (Trabasso y Sperry, 1985), segmentaron la transcripción para identificar conexiones causales entre los enunciados de los locutores. Los resultados indicaron que los enunciados eran más recordados e incluidos en las respuestas cuanto mayor fuera su número de conexiones causales, independientemente de la modalidad en que fueran presentados. A fin de examinar el rol de los enunciados que introducían los temas de conversación, se llevó a cabo un re-análisis de los datos (Cevasco, 2010). Los resultados indicaron que aquellos enunciados que marcaban un cambio de tema y poseían un número alto de conexiones causales eran los más recordados, y los que no generaban un cambio de tema y no tenían tantas conexiones eran los menos recordados.

Otro estudio (Cevasco, 2009) investigó el procesamiento de conexiones causales y de marcadores del discurso adversativos. Los marcadores del discurso adversativos indican un contraste entre dos enunciados adyacentes. Para estudiar el rol de "pero", un grupo de universitarios escuchó secciones de conversaciones, y al observar una oración en pantalla, debió responder si los ayudaba a entender el último enunciado escuchado. La oración presentada estaba causalmente conectada con este último. Se observó que, cuando el marcador precedía la escucha del enunciado, los sujetos respondían más rápidamente a la oración que cuando no lo hacía. Estos resultados sugieren que "pero" facilita la integración de enunciados adyacentes, y así el establecimiento de conexiones causales entre enunciados no adyacentes. Fox Tree y Schrock (1999) encontraron a su vez que "oh" facilita la integración de enunciados escuchados.

Estos estudios nos permiten sugerir que la conectividad causal, los enunciados que introducen temas de conversación, y la presencia de marcadores del discurso tienen un rol en la comprensión del discurso oral no expositivo, dejando abierta la pregunta acerca de cuál es su rol en la comprensión del discurso oral expositivo.

\section{Comprensión del discurso oral expositivo}

A continuación, se describirán estudios acerca de la comprensión de discurso oral expositivo presentado durante el dictado de clases, por parte de estudiantes universitarios no nativos. Si bien estos estudios no han considerado la compren- 
sión de estudiantes nativos, sus resultados pueden resultar un punto de partida para poner a prueba si también resultarían facilitadores para ellos, y quizás aún más, en el caso de estudiantes pre-universitarios.

Flowerdew y Tauroza (1995) examinaron el rol de los marcadores del discurso (ok-bueno-y-pero-ahora-entonces-porque) en la comprensión de clases de Ingeniería en inglés videograbadas, por parte de estudiantes universitarios chinos. Presentaron a un grupo la versión original, la cual incluía todos los marcadores que producidos espontáneamente por el profesor, y a otro una versión en la que los marcadores habían sido recortados. Encontraron que el grupo que había observado la clase que contenía los marcadores originales proveía mejores resúmenes, y respondía mejor a preguntas que el que había recibido la clase sin los marcadores. Jung (2003) observó a su vez que universitarios coreanos mostraban un mejor recuerdo de una clase en inglés cuando había sido presentada con marcadores que sin ellos.

El rol de la velocidad de dicción del profesor también fue explorado. Robinson, Sterling, Skinner, y Robinson (1997) encontraron que universitarios nativos de inglés respondían mejor a preguntas de comprensión, y consideraban más importante el tema de una clase de Biología grabada en ese idioma, cuando era presentada a una velocidad de dicción lenta (100 palabras-por-minuto), que normal (150-palabras por minuto) o rápida (200 palabras-por-minuto). Hayati (2010) encontró que universitarios iraníes que escuchaban clases en inglés a una velocidad de dicción natural o lenta respondían mejor a preguntas de comprensión en inglés después de tomar las clases que antes, pero la mejora era mayor cuando las habían escuchado a una velocidad natural que lenta. Es decir, a pesar de que se asume que cuando más lenta es la velocidad de presentación, más se facilita la comprensión, es posible también implique una pérdida de la naturalidad del discurso.

Un tercer tema explorado ha sido el efecto del entrenamiento en habilidades metacognitivas. Éstas involucran el conocimiento acerca del propio aprendizaje, y cómo favorecerlo. Vandergrift and Tafaghodtari (2010) propusieron que los estudiantes pueden ser entrenados para: 1) establecer, antes de una clase, predicciones acerca de los posibles temas y sub-temas que tratará (planificación), 2) durante la clase, comparar sus predicciones con la información que se presenta, y corregirla o ampliarla (monitorización) 3) finalizada la clase, comparar su comprensión y notas con la de sus compañeros, para agregar/corregir información (evaluación), y 4) establecer metas y objetivos para la siguiente clase. Utilizando este entrenamiento, los autores encontraron una facilitación del recuerdo por parte de estudiantes no nativos de francés que escucharon clases grabadas en ese idioma, en comparación con estudiantes que no lo recibieron. Selamat y Sidhu (2011) encontraron que este entrenamiento aumentaba la percepción de estudiantes no nativos de inglés acerca de sus capacidades de comprensión, y de extraer información de las clases. Resultados similares fueron encontrados por Rasouli et al. (2013).
Estos estudios nos brindan información acerca del rol facilitador que puede tener que el profesor introduzca marcadores del discurso entre los enunciados, que dicte la clase a una velocidad natural, y que promueva en sus estudiantes la utilización de habilidades metacognitivas. Sin embargo, continúa abierta la pregunta por el establecimiento de conexiones entre enunciados, tales como las causales, en la comprensión del discurso oral expositivo.

\section{Conclusiones}

El objetivo de este trabajo fue tanto destacar los aportes que los estudios acerca de la comprensión del discurso escrito expositivo y el discurso oral no expositivo y expositivo pueden hacer al dictado de clases, así como proponer la importancia de realizar nuevos estudios que exploren las variables que facilitan la comprensión del discurso escrito expositivo, y no se han investigado en relación con el discurso oral expositivo.

Los estudios acerca de la comprensión del discurso escrito expositivo sugieren que es posible introducir cambios en un material didáctico para facilitar su comprensión, tanto de estudiantes pre-universitarios como universitarios. Por ejemplo, se puede promover que el lector vea co-activado su conocimiento previo y el que el texto brinda, a través del diseño de textos de refutación. A su vez, el formato del texto puede ser editado a fin de que las oraciones siempre compartan términos, y que tanto oraciones adyacentes como distantes estén conectadas, ya que estas revisiones promueven que los estudiantes recuerden mejor, y respondan mejor a preguntas acerca del texto.

Los estudios acerca de la comprensión del discurso oral no expositivo nos brindan información acerca de los procesos que están en juego cuando se expone discurso oral, ya sea expositivo o no, teniendo en cuenta que, tanto un hablante que da instrucciones como un profesor que dicta una clase producen errores del habla, y proveen claves prosódicas. Sus resultados sugieren que los oyentes ven demorada su comprensión cuando los hablantes corrigen un enunciado, pero no cuando repiten palabras. A su vez, las pausas marcadas preparan para procesar información novedosa o difícil. En cuanto a claves prosódicas, éstas permiten que los oyentes segmenten los enunciados, y se enfoquen en las palabras que introducen nueva información. Por otra parte, los enunciados que tienen varias conexiones causales, aquellos que introducen temas y la presencia de marcadores del discurso facilitan la comprensión de conversaciones.

Los estudios acerca de la comprensión del discurso oral expositivo sugieren que la inclusión de marcadores del discurso por parte del profesor, tanto como la utilización de una velocidad de dicción normal, no muy rápida pero tampoco muy lenta, y el entrenamiento de los estudiantes en la utilización de habilidades metacognitivas facilitan el recuerdo y respuesta a preguntas acerca de una clase.

Considerados conjuntamente, estos estudios nos brindan interesante información acerca de la comprensión del discurso. Sin embargo, a pesar de que los estudios acerca de la compren- 
sión del discurso expositivo escrito resaltan la importancia de establecer conexiones entre enunciados, los estudios acerca de la comprensión del discurso oral no expositivo y expositivo no han brindado aún información acerca de este tema, por lo que resulta relevante proponer que sean investigados en el futuro. Realizar estudios acerca de este tema nos permitiría establecer, por ejemplo, si la co-activación de enunciados que se refieran al conocimiento previo incorrecto de los estudiantes y al conocimiento correcto, o el agregado de enunciados que permitan establecer nuevas conexiones tendrían un efecto facilitador en la comprensión, y si éste sería similar en el ámbito pre-universitario y universitario. Establecer el efecto de la presentación conjunta de ideas erróneas y correctas en forma oral es importante, dado que la posesión de conocimiento incorrecto parece perjudicar la comprensión de lectores tanto universitarios, como pre-universitarios, y éste podría también ocurrir a los oyentes, quienes se encuentran a su vez bajo la presión de procesar el discurso mientras es producido. Es decir, el profesor puede explicitar las ideas incorrectas que suelen tener los estudiantes para contrastarlas con las correctas, como lo haría el autor de un texto de refutación. Sin embargo, lo hará a una velocidad de dicción determinada, y produciendo errores del habla y claves prosódicas. En consecuencia, el procesamiento de errores del habla podrá demorar la detección de la inconsistencia entre las ideas correctas e incorrectas, requiriendo que el profesor produzca estos enunciados a una velocidad de dicción natural, pero más baja que otros, ya que la posesión de conocimiento previo incorrecto parece resultar en un procesamiento más lento de las oraciones que lo refutan. El profesor podrá también valerse de la capacidad de interactuar con los estudiantes, para evaluar si están pudiendo reemplazar su conocimiento incorrecto por el correcto, especialmente cuando se trata de estudiantes preuniversitarios. Esto le permitirá determinar si necesita repetir información, acentuar palabras, o agregar enunciados que promuevan el establecimiento de nuevas conexiones. Del mismo modo, la inclusión de marcadores del discurso entre los enunciados puede facilitar su integración, así como la introducción de enunciados que marquen claramente los cambios de tema puede promover el recuerdo de los tópicos tratados.

Cabe destacar que el profesor puede además entrenar en la utilización de estrategias metacognitivas, tanto para facilitar el aprendizaje individual, como grupal a través de que los estudiantes compartan sus notas una vez finalizadas las clases. Si bien los estudios descritos se han enfocado en la comprensión de estudiantes universitarios, dado que los estudiantes pre-universitarios están desarrollando sus capacidades de estudio y toma de notas, este tipo de entrenamiento puede resultar aún más facilitador. Es decir, puede contribuir al desarrollo temprano del uso de estas estrategias, y a su vez favorecer el aprendizaje cooperativo.

En conclusión, el estudio de la comprensión del discurso oral expositivo es un tema que se encuentra abierto, y futuras investigaciones pueden valerse de lo que sabemos acerca del discurso escrito expositivo y el discurso oral no expositivo y expositivo para facilitar el aprendizaje de los estudiantes que participan de una clase.

\section{Referencias}

1. Ariasi, N. y Mason, L. (2011). Uncovering the effect of text structure in learning from a science text: An eye-tracking study. Instructional Science, 39, 581-601. http://dx.doi. org/10.1007/s11251-010-9142-5

2. Barr, D. y Seyfeddinipurr, M. (2010). The role of fillers in listener attributions for speaker disfluency. Language and Cognitive Processes, 25, 441-455. http://dx.doi. org/10.1080/01690960903047122

3. Barreyro, J.P., Molinari Marotto, C., Bechis, S. y Cevasco, J. (2012). Comprensión de textos expositivos y métodos sistemáticos de revisión de textos: el efecto del incremento de la densidad de relaciones y la repetición de términos. Investigaciones en Psicología, 17, 9-24.

4. Beck, I. L., McKeown, M. G., Sinatra, G. M. y Loxterman, J. A. (1991). Revising social studies text from a text-processing perspective: Evidence of improved comprehensibility. Reading Research Quarterly, 26, 251-276. http://dx.doi. org/10.2307/747763

5. Bögels, S., Schriefers, H., Vonk, W., Chwilla, D. J. y Kerkhofs, R. (2010). The interplay between prosody and syntax in sentence processing: The case of subject- and object-control verbs. Journal of Cognitive Neuroscience, 22, 1036-1053. http://dx.doi.org/10.1162/jocn.2009.21269

6. Brennan, S.E. y Schober, M.F. (2001). How listeners compensate for disfluencies in spontaneous speech. Journal of Memory and Language 44, 274-296. http://dx.doi. org/10.1006/jmla.2000.2753

7. Britton, B.K. y Gulgoz, S. (1991). Using Kintsch's computational model to improve instructional text: Effects of repairing inference calls on recall and cognitive structures. Journal of Educational Psychology, 83, 329-345. http:// dx.doi.org/10.1037/0022-0663.83.3.329

8. Broughton, S. H., Sinatra, G. M., y Reynolds, R. E. (2010). The nature of the refutation text effect: An investigation of attention allocation. The Journal of Educational Research, 103, 407-423. http://dx.doi. org/10.1080/00220670903383101

9. Cevasco, J. (2009). The role of connectives in the comprehension of spontaneous spoken discourse. The Spanish Journal of Psychology, 12, 56-65. http://dx.doi. org/10.1017/S1138741600001475

10. Cevasco, J. (2010). El interjuego entre los temas de conversación y la conectividad causal de los enunciados conversacionales en el recuerdo del discurso oral espontáneo. Perspectivas en Psicología, 7, 1-7.

11. Cevasco, J. y van den Broek, P. (2008). The importance of causal connections in the comprehension of spontaneous spoken discourse. Psicothema, 20, 801-806. 
12. Chafe, W. (1994). Discourse, Consciousness and Time. Chicago: University of Chicago Press.

13. Corley, M. y Hartsuiker, R. J. (2011). Why um helps auditory word recognition: The temporal delay hypothesis. PLoS ONE, 6, e19792. http://dx.doi.org/10.1371/journal. pone.0019792

14. Cromley, J.G., Snyder-Hogan, L.E. y Luciw-Dubas, U.A. (2010). Reading comprehension of scientific text: A domain-specific test of the direct and inferential mediation model of reading comprehension. Journal of Educational Psychology, 102, 687-700. http://dx.doi.org/10.1037/ $\underline{\mathrm{a} 0019452}$

15. Diakidoy, I. N., Kendeou, P. y Ioannides, C. (2003). Reading about energy: The effects of text structure in science learning and conceptual change. Contemporary Educational Psychology, 28, 335-356. http://dx.doi.org/10.1016/ S0361-476X(02)00039-5

16. Ferreira, F. y Anes, M. (1994). Why study spoken language processing? In M. Gernsbacher (Ed.), Handbook of Psycholinguistics (pp. 33-56). San Diego, CA: Academic Press.

17. Flowerdew, J. y Tauroza, S. (1995). The effect of discourse markers on second language lecture comprehension. Studies in Second Language Acquisition, 17, 435-458. http:// dx.doi.org/10.1017/S0272263100014406

18. Fox Tree, J.E. (1995). The effects of false starts and repetitions on the processing of subsequent words in spontaneous speech. Journal of Memory and Language, 34, 709-738. http://dx.doi.org/10.1006/jmla.1995.1032

19. Fox Tree, J. E. y Schrock, J.C. (1999). Discourse markers in spontaneous speech: Oh what a difference an oh makes. Journal of Memory and Language, 40, 280-295. http:// dx.doi.org/10.1006/jmla.1998.2613

20. Fraundorf, S.H. y Watson, D.G. (2011). The disfluent discourse: Effects of filled pauses on recall. Journal of Memory and Language, 65, 161-175. http://dx.doi.org/10.1016/j. jml.2011.03.004

21. Fraundorf, S.H., Watson, D.G. y Benjamin, A.S. (2012). The effects of age on the strategic use of pitch accents in memory for discourse: a processing-resource account. Psychology and Aging, 27, 88-98. http://dx.doi.org/10.1037/ a0024138

22. Gilabert, R., Martinez, G. y Vidal-Abarca, E. (2005). Some good texts are always better: text revision to foster inferences of readers with high and low prior background knowledge. Learning and Instruction, 15, 45-68. http:// dx.doi.org/10.1016/j.learninstruc.2004.12.003

23. Gunraj, D. y Klin, C. M. (2012). Hearing story characters' voices: auditory imagery during reading. Discourse Processes, 49, 137-153. http://dx.doi.org/10.1080/01638 53X.2012.657755

24. Hayati, A. (2010). The effect of speech rate on listening comprehension of EFL learners. Creative Education, 1, 107-114. http://dx.doi.org/10.4236/ce.2010.12016
25. Ito, K. y Speer, S.R. (2008). Anticipatory effects of intonation: eye movements during instructed visual search, Journal of Memory and Language 58, 541-73. http:// dx.doi.org/10.1016/j.jml.2007.06.013

26. Jung, E.H. (2003). The role of discourse signaling cues in second language listening comprehension. Modern Language Journal, 87, 562-577. http://dx.doi. org/10.1111/1540-4781.00208

27. Kendeou, P., Muis, K.R. y Fulton, S. (2011). Reader and text factors in reading comprehension processes. Journal of Research in Reading, 34, 365-383. http://dx.doi. org/10.1111/j.1467-9817.2010.01436.x

28. Kendeou, P. y van den Broek, P. (2005). The role of readers' misconceptions on comprehension of scientific text. Journal of Educational Psychology, 97, 235-245. http://dx.doi. org/10.1037/0022-0663.97.2.235

29. Kendeou, P. y van den Broek, P. (2007). The effects of prior knowledge and text structure on comprehension processes during reading of scientific texts. Memory y Cognition, 35, 1567-1577. http://dx.doi.org/10.3758/BF03193491

30. Kjelgaard, M.M. y Speer, S.R. (1999). Prosodic facilitation and interference in the resolu- tion of temporary syntactic closure ambiguity. Journal of Memory and Language, 40, 153-194. http://dx.doi.org/10.1006/jmla.1998.2620

31. Kintsch, W. y van Dijk, T.A. (1978). Toward a model of text comprehension and production. Psychological Review, 85, 363-394. http://dx.doi.org/10.1037/0033-295X.85.5.363

32. Kraljic, T. y Brennan, S. (2005). Prosodic disambiguation of syntactic structure: For the speaker or for the addressee? Cognitive Psychology, 50, 194 -231. http://dx.doi. org/10.1016/j.cogpsych.2004.08.002

33. Ladd, D.R. (1996). Intonational phonology. Cambridge: Cambridge University Press.

34. MacGregor, L.J., Corley, M. y Donaldson, D.I. (2010). Listening to the sound of silence: Disfluent silent pauses in speech have consequences for listeners. Neuropsychologia, 48, 3982-3992. http://dx.doi.org/10.1016/j.neuropsychologia.2010.09.024

35. Mason, L., Gava, M. y Boldrin, A. (2008). On warm conceptual change: The interplay of text, epistemological beliefs, and topic interest. Journal of Educational Psychology, 100, 291-309. http://dx.doi.org/10.1037/0022-0663.100.2.291

36. McCloskey, M. (1982). Naive conceptions of motion (No. ED 223 415). Baltimore, MD: John Hopkins University, Department of Psychology.

37. McCrudden, M. T.(2012). Readers'use of online discrepancy resolution strategies. Discourse Processes, 49, 107-136. http://dx.doi.org/10.1080/0163853X.2011.647618

38. Molinari Marotto, C., Barreyro, J.P., Cevasco, J. y van den Broek, P. (2011). Generation of emotional inferences during text comprehension: Behavioral data and implementation through the landscape model. Escritos de Psicología, 4, 9-7.

39. Mulder, G. y Sanders, T.J.M. (2012). Causal coherence 
relations and levels of discourse representation. Discourse Processes, 49, 501-522. http://dx.doi.org/10.1080/01638 $\underline{53 X .2012 .692655}$

40. Ratebi, Z. y Amirian, Z. (2013). The use of metacognitive strategies in listening comprehension by Iranian university students majoring in English: a comparison between high and low proficient listeners. Journal of Studies in Education, 3, 140-154. http://dx.doi.org/10.5296/jse.v3i1.2969

41. Rasouli, M., Mollakhan, K. y Karbalaei, A. (2013). The effect of metacognitive listening strategy training on listening comprehension in Iranian EFL context. European Online Journal of Natural and Social Sciences, 2, 115-128.

42. Robinson, S.L., Sterling, H.E., Skinner, C.H. y Robinson, D.H. (1997). Effects of lecture rate on students' comprehension and ratings of topic importance. Contemporary Educational Psychology, 22, 260-267. http://dx.doi. org/10.1006/ceps.1997.0929

43. Schafer, A.J., Speer, S., Warren, P. y White, S.D. (2000). Intonational disambiguation in sentence production and comprehension. Journal of Psycholinguistic Research, 29, 169-182. http://dx.doi.org/10.1023/A:1005192911512

44. Sedivy, J., M. Tanenhaus, C. Chambers y Carlson, G. (1995). Using intonationally-marked presuppositional information in on-line language processing: evidence from eye movements to a visual model. Proceedings of the 17th Annual Conference of the Cognitive Science Society, ed. por J.D. Moore and J.F. Lehman, 375-80. Hillsdale: Erlbaum.

45. Selamat, S. y Sidhu, G. K. (2011). Student perceptions of metacognitive strategy use in lecture listening comprehension, Language Education in Asia, 2, 185-198. http:// dx.doi.org/10.5746/LEiA/11/V2/I2/A02/Selamat_Sidhu

46. Snedeker, J. y Trueswell, J. (2003). Using prosody to avoid ambiguity: Effects of speaker awareness and referential context. Journal of Memory and Language, 48, 103-130.

47. Toepel, U., Pannekamp, A. y Alter, K. (2007). Catching the news: Processing strategies in listening to dialogs as measured by ERPs. Behavioral and Brain Functions, 3:53.

48. Trabasso, T. y Sperry, L.L. (1985). Causal relatedness and importance of story events. Journal of Memory and Language, 24, 595-611.

49. Van den Broek, P. (2010). Using texts in science education: cognitive processes and knowledge representation. Science, $328,453-456$.
50. Van den Broek, P. y Kendeou, P. (2008). Cognitive processes in comprehension of science texts: The role of co-activation in confronting misconceptions. Applied Cognitive Psychology, 22, 335-351. http://dx.doi.org/10.1002/ acp. 1418

51. Vandergrift, L. y Tafaghodtari, M. (2010). Teaching L2 learners how to listen does make a difference: an empirical study. Language Learning, 60, 470-467. http://dx.doi. org/10.1111/j.1467-9922.2009.00559.x

52. Vidal-Abarca, E., Gilabert, R. y Abad, N. (2002). Una propuesta para hacer buenos textos expositivos: Hacia una tecnología del texto. Infancia y Aprendizaje, 25, 499-514. http://dx.doi.org/10.1174/021037002762064064

53. Vidal-Abarca, E., Martínez, G. y Gilabert, R. (2000). Two procedures to improve instructional text: Effects on memory and learning. Journal of Educational Psychology, 92, 107-116.

54. Vidal-Abarca, E., Reyes, H., Gilabert, R., Calpe, J., Soria, E. y Graesser, A.C. (2002). ETAT: expository text analysis tool. Behavior Research Methods, Instruments and Computers, 34, 93-107.

55. Wagner, M. y Watson, D.G. (2010). Experimental and theoretical advances in prosody: A review. Language and Cognitive Processes, 25, 905-945.

56. Weber, A., Braun, B. y Crocker, M.W. (2006). Finding referents in time: eye-tracking evidence for the role of contrastive accents. Language and Speech, 49, 367-92.

57. Zwaan, R.A. y Rapp, D.N. (2006). Discourse comprehension. En M. Traxler y M. A. Gernsbacher (Ed.), Handbook of psycholinguistics, 2nd ed. (pp. 725-764). San Diego, CA: Academic Press.

Fecha de recepción: 29 de enero de 2013 Fecha de recepción de la versión modificada: 23 de abril de 2013 Fecha de aceptación: 19 de junio de 2013 